\title{
Acute postpartum pulmonary edema in a 23-year-old woman 5 days after cesarean delivery
}

\author{
Caitlin Dunne, MD;* Antonio Meriano, BSc, MD, Dip Sport Med ${ }^{\dagger+}$
}

\begin{abstract}
The presentation of acute dyspnea after pregnancy is rare, but should bring a number of important conditions to the mind of the attending physician. Pulmonary embolism, amniotic fluid embolism, pneumonia, aspiration and pulmonary edema are some of the potentially devastating causes that must be considered. The percentage of pregnancies that are complicated by acute pulmonary edema has been estimated to be $0.08 \%$. The most common contributing factors include the administration of tocolytic agents, underlying cardiac disease, iatrogenic fluid overload and preeclampsia. No matter what the underlying pathology, prompt administration of appropriate resuscitation is always the first priority. Only after the patient has been stabilized can attention be turned to diagnosis and specific treatment. This case examines one such presentation and reviews some of the diagnostic possibilities.
\end{abstract}

Keywords: emergency medicine, pulmonary edema, obstetric emergencies

\section{RÉSUMÉ}

Les cas de dyspnée aiguë après une grossesse sont rares, mais ils devraient évoquer, pour le médecin traitant, un certain nombre d'affections sous-jacentes. Parmi les causes potentiellement dévastatrices qui doivent être envisagées, citons une embolie pulmonaire, une embolie amniotique, une pneumonie, une aspiration et un œdème pulmonaire. Le pourcentage de grossesses compliquées par un œdème pulmonaire aigu a été évalué à $0,08 \%$. Les facteurs contributifs les plus courants incluent l'administration de tocolytiques, une cardiopathie sous-jacente, une surcharge liquidienne iatrogène et une prééclampsie. Quelle que soit la pathologie sous-jacente, la mise en œuvre rapide de mesures de réanimation appropriées demeure la priorité. Le diagnostic et la prise en charge ne doivent être envisagés qu'après avoir stabilisé la patiente. Nous présentons un tel cas et passons en revue quelques diagnostics possibles.

\section{Introduction}

Pulmonary edema of cardiac origin is a common medical condition that can range in severity from chronic, to subclinical, to acute, with accompanying severe respiratory compromise. Resuscitation is the foremost priority, fol- lowed by formulation of a differential diagnosis to address the underlying condition. In a young, previously healthy postpartum patient, the differential must be expanded to include some less prevalent causes, such as peripartum cardiomyopathy and cardiac failure secondary to tocolytics. A clinician needs to be aware of the pertinent physiologic

\footnotetext{
*Resident, Department of Obstetrics and Gynaecology, University of British Columbia, Vancouver, BC, +Medical Director, Department of Emergency Medicine, Windsor Regional Hospital, Windsor, Ont., $¥$ Academic Director, Emergency Medicine, South Western Ontario Medical Education Network, Schulich School of Medicine, University of Western Ontario, London, Ont.
}

Submitted Jan. 2, 2008; Revised Jun. 6, 2008; Accepted Jul. 24, 2008

This article has been peer reviewed.

CJEM 2009;11(2):178-81 
differences that accompany pregnancy, such as increased cardiac output, expansion of blood volume, physiologic anemia and decreased systemic vascular resistance. In this case, a postpartum patient presented with acute pulmonary edema and severe respiratory compromise. A discussion of the management and workup of acute respiratory distress in the postpartum patient is included below.

\section{Case report}

A 23-year-old white woman with acute dyspnea was 5 days postpartum from her first pregnancy when she arrived by ambulance to the emergency department (ED). Her only previous respiratory complaint was a mild cough the preceding day. She had been discharged from hospital 1 day before presentation, after a 5-day admission post-cesarean delivery that was complicated by postpartum pregnancy-induced hypertension. Her baby had been delivered at 39 weeks and 5 days gestation after 22 hours of labour, which failed to progress to a spontaneous vaginal delivery. The discharge medications included labetalol $200 \mathrm{mg}, 3$ times daily, and ferrous gluconate. She had no remarkable medical history or significant allergies, and she denied tobacco, alcohol and drug use. She had no family history of cardiac or respiratory disease.

The patient reported a 1-day history of mild coughing that progressed to severe shortness of breath and chest heaviness upon waking from a nap. In the ED she looked pale, unwell and was only able to speak in 1-2 word sentences. She denied visual changes, headache, hand and face swelling or right upper-quadrant pain. Her vital signs included a temperature of $37.7^{\circ} \mathrm{C}$, her oxygen saturation was $82 \%$ on room air, her heart rate was 132 beats/min, her blood pressure was $160 / 90 \mathrm{~mm} \mathrm{Hg}$ and her respiratory rate was 24 breaths/min. Breath sounds were decreased, crackles were present bilaterally at the lung bases, and peripheral edema was noted. The jugular venous pressure could not be evaluated because of body habitus. The rest of the physical examination was unremarkable.

An electrocardiogram revealed sinus tachycardia, and her chest radiograph illustrated bilateral pulmonary edema. Her urinalysis showed trace amounts of intact blood and was negative for glucose, ketones, nitrites, leukocytes and protein. Immediate echocardiography showed normal ventricular function (i.e., the ejection fraction was $65 \%$ ), trace mitral and tricuspid regurgitation, normal wall motion and no pericardial effusion, clots or shunts. An arterial blood gas test revealed that the $\mathrm{pH}$ was 7.42, the partial pressure of carbon dioxide was $39 \mathrm{~mm} \mathrm{Hg}$, the partial pressure of oxygen was $43 \mathrm{~mm} \mathrm{Hg}$ and the bicarbonate was $25 \mathrm{mmol} / \mathrm{L}$ on $100 \%$ oxygen.
Initial resuscitative measures were instituted in the ED, and included oxygen administration by nonrebreather mask, which increased her oxygen saturation to $94 \%$. Additional measures included bilevel positive airway pressure at $16 / 5 \mathrm{~cm} \mathrm{H}_{2} \mathrm{O}$ with a fraction of inspired oxygen $\left(\mathrm{FiO}_{2}\right)$ of $80 \%$. Intravenous heparin was administered, as a pulmonary embolism could not be ruled out. Nitroglycerin was also started intravenously and titrated up to $140 \mu \mathrm{g} / \mathrm{h}$. Captopril $25 \mathrm{mg}$ was given sublingually. Furosemide was administered intravenously at a dose of $40 \mathrm{mg}$. After 2 hours of treatment the patient had diuresed $1600 \mathrm{~mL}$ of urine. She showed considerable improvement in her respiratory status and her $\mathrm{FiO}_{2}$ decreased to $70 \%$.

The patient was admitted to the coronary care unit. Further investigations included spiral chest computed tomography, which was negative for pulmonary embolism or airspace disease; normal thyroid-stimulating hormone and parathyroid hormone tests; and normal repeat echocardiography. Her urine was negative for vanillylmandelic acid and catecholamines. Blood cultures were negative.

After 2.5 days, her pulmonary edema was largely resolved and her oxygen saturation was $98 \%$ on room air. She had no dyspnea, chest pain, headache or visual changes. Her blood pressure was $153 / 77 \mathrm{~mm} \mathrm{Hg}$ despite the administration of metoprolol and lisinopril. The patient continued to improve and was discharged after 7 days. Her discharge medications included metoprolol, amlodipine, hydrochlorothiazide and lisinopril.

\section{Discussion}

The differential diagnoses for postpartum dyspnea include the following:

without pulmonary edema

- pulmonary embolism, amniotic fluid embolism, pneumonia, foreign body aspiration, psychogenic dyspnea with pulmonary edema

- cardiogenic: peripartum cardiomyopathy, preeclampsia-related heart failure, underlying cardiac disease (e.g., valvulopathy), myocardial ischemia and sepsis with poor cardiac output

- noncardiogenic: iatrogenic fluid overload, thyroid disease, tocolytic therapy or medication-related sepsis, acute respiratory distress syndrome

In this case, a number of the aforementioned diagnoses were plausible. However, the results of subsequent investigations appeared to rule out pulmonary embolism, amniotic fluid embolism, pneumonia and sepsis. Ischemic heart disease was ruled out as there was no evidence of abnormal cardiac markers or abnormalities on the echocardio- 
gram. Careful review of the patient's chart revealed no tocolytic therapy.

Postpartum cardiomyopathy (PPCM) is a rare entity, but a mortality rate of $50 \%$ has been suggested. ${ }^{1}$ The cause of this form of cardiomyopathy is unknown. It is defined by 4 criteria: $^{1}$

1) absence of an identifiable cause of pulmonary edema

2) development of cardiac failure within the last month of pregnancy or within 5 days of delivery

3) absence of recognizable cardiac disease before the last month of pregnancy

4) left ventricular systolic dysfunction demonstrated on echocardiogram

The actual incidence of the illness varies largely by geographic region, with an incidence of 1 out of 15000 people in the United States and 1 out of 100 people in Zaira, Nigeria. ${ }^{2,3}$ The cause of PPCM remains unknown, but a number of risk factors have been proposed: ${ }^{4}$

- age greater than 30 years

- multiparity

- African descent

- pregnancy with multiple fetuses

- a history of preeclampsia, eclampsia or postpartum hypertension

- a history of maternal cocaine use

Postpartum cardiomyopathy most commonly presents with dyspnea, but other presenting complaints may include cough, orthopnea, paroxysmal nocturnal dyspnea and chest pain. ${ }^{1}$ Echocardiography should be performed in all patients with suspected PPCM. Echocardiographic findings will demonstrate left ventricular enlargement, mitral and tricuspid regurgitation and possibly a small pericardial effusion. ${ }^{5}$ Women with documented PPCM should be warned that there is a high risk of subsequent cardiac deterioration and death with any subsequent pregnancy. ${ }^{6}$ In our particular case, 2 separate echocardiograms demonstrated normal left ventricular function. This fact, along with any other diagnostic criteria, made PPCM highly unlikely in our patient.

Preeclampsia is an illness that is characterized by the presence of proteinuria and hypertension occurring after the 20th week of gestation; edema may also be present, although it is not essential for diagnosis. The patient is considered to have eclampsia if seizures develop. ${ }^{7}$ The patient with preeclampsia/eclampsia may develop refractory hypertension, neurologic dysfunction, renal failure, liver rupture or failure, hemolytic anemia, elevated liver enzymes and low platelet count syndrome, pulmonary edema, and/or disseminated intravascular coagulation, which is usually associated with concomitant placental abruption. The pathogenesis of pulmonary edema associated with preeclampsia and eclampsia is felt to be multifactorial. ${ }^{8}$ The incidence of pulmonary edema may be as high as $3 \%$ with this illness. ${ }^{8}$ Excessive elevations in pulmonary vascular hydrostatic pressure compared with plasma oncotic pressure may produce pulmonary edema in some women, particularly during the postpartum period. However, not all preeclamptic patients with pulmonary edema demonstrate this phenomenon. ${ }^{8}$ The treatment of preeclampsia- and eclampsia-related pulmonary edema is largely supportive and should include supplemental oxygen and fluid restriction. Definitive treatment is delivery of the fetus when the mother is stabilized, which usually leads to resolution of the symptoms within 48 hours. Diuretics should be administered only in the presence of clinically significant fluid overload. Consideration of actual circulating volume and the possibility of reducing placental perfusion should be kept in mind. Our patient was diagnosed with pregnancyinduced hypertension, but did not meet the case definition of preeclampsia, as she did not have proteinuria (i.e., her 24-hour urine sample was negative) and had no pre- or postnatal record of proteinuria.

In one case series, 26 of the 37 patients developed pulmonary edema postpartum (at a mean of $71 \mathrm{~h}$ after delivery), and 4 patients died. ${ }^{9}$ Older and multigravid patients appeared to be at increased risk of pulmonary edema in this setting. Pulmonary edema was often associated with the dysfunction of other systems. Of the 37 patients, 18 had disseminated intravascular coagulation, 10 had acute renal failure, 6 experienced a hypertensive crisis, 5 had a cardiopulmonary arrest and 2 had cerebral edema. ${ }^{9}$

Amniotic fluid embolism is a rare but potentially fatal outcome of pregnancy. The classic presentation involves the acute onset of severe dyspnea, hypoxemia and hypotension, followed within minutes by cardiac arrest. It usually occurs during labour and delivery ${ }^{10}$ although it has been reported following uterine trauma and abortions. If patients survive, respiratory failure and disseminated intravascular coagulation frequently develop. The patient typically has a sudden deterioration in her condition and may arrest. One study of 46 patients found a dismal maternal mortality rate of $61 \%$, with neurologically intact survival seen in $15 \%$ of women. ${ }^{10}$ In the same paper, the authors felt that cases of amniotic fluid embolism posed similar characteristics to an anaphylactic reaction, suggesting the actual pathology may be a severe allergic reaction. True confirmation of the diagnosis can be complicated and controversial. Using a Swan-Ganz catheter to obtain fetal squamous cells from the pulmonary artery was formerly felt to be the true gold standard. ${ }^{11,12}$ However, the specificity of this finding is unclear, as squamous cells can be 
found in the maternal pulmonary circulation in the absence of other clinical findings of amniotic fluid embolism. ${ }^{12} \mathrm{~A}$ report by Vanmaele and colleagues ${ }^{13}$ emphasizes the role of pulmonary artery catheterization to diagnose and monitor obstetric patients with respiratory compromise and left ventricular dysfunction. The diagnosis is usually made, however, on the basis of clinical signs and symptoms. In our patient's case, a Swan-Ganz catheter was not used. However, we feel that the onset 5 days postdelivery, the absence of hypotension, the rapid response to vasodilation therapy and the lack of left ventricular dysfunction on her echocardiogram made the diagnosis of amniotic fluid embolism extremely unlikely.

Iatrogenic fluid overload is another possible cause, and a review of our patient's labour and delivery record revealed significant fluid administration. Over the course of her 22 hours of labour, she received approximately $5500 \mathrm{~mL}$ of normal saline. This corresponds to an average fluid administration of $250 \mathrm{~mL} / \mathrm{h}$. If we consider that our patient would have required approximately $125 \mathrm{~mL} / \mathrm{h}$ for maintenance, plus extra fluid administration for deficits, insensible losses from vomiting during labour and delivery, and surgical losses because of blood loss and evaporative loss (500$1000 \mathrm{~mL}$ of blood loss and $6 \mathrm{~mL} / \mathrm{kg} / \mathrm{h}$ of evaporative loss), an estimate of our patient's fluid requirements would be approximately $3000-4000 \mathrm{~mL}$. We do not have a record of her oral fluid intake during this time period. Sciscione and colleagues ${ }^{14}$ reviewed 10 years of pregnancy data to examine pulmonary edema in pregnancy. Of the 20 patients in their study with postpartum pulmonary edema, the most common attributable cause was iatrogenic fluid overload (patients had a positive fluid balance of $6022 \mathrm{~mL}$, standard deviation $3340 \mathrm{~mL}$, in the preceding $48 \mathrm{~h}$ before diagnosis). Although a record of true intake or outtake fluid was not kept for this patient, her positive fluid balance is estimated to be somewhat less than seen in this review. We cannot rule out iatrogenic fluid overload as a contributing factor; however, the absence of tocolytic therapy and her presentation 5 days postpartum reduces the likelihood that volume overload was the sole source of her pathology.

\section{Conclusion}

The presentation of acute pulmonary edema in a previously healthy young woman who has recently given birth is an uncommon clinical scenario with some potentially fatal complications. Initial resuscitation and investigation should take into consideration multiple causative possibilities.
Positive pressure ventilation and symptomatic support will often allow enough time to identify a probable cause.

Competing interests: None declared.

\section{References}

1. Demakis JG, Rahimtoola SH, Sutton GC, et al. Natural course of peripartum cardiomyopathy. Circulation 1971;44:1053-61.

2. Cunningham FG, Pritchard JA, Hankins GD, et al. Peripartum heart failure: Idiopathic cardiomyopathy or compounding cardiovascular events? Obstet Gynecol 1986;67:157-68.

3. Sliwa K, Damasceno A, Mayosi BM. Epidemiology and etiology of cardiomyopathy in Africa. Circulation 2005;112:3577-83.

4. Quinn B. Doyle, McInerney J. Postnatal precordial pain. Pulmonary embolism or peripartum cardiomyopathy. Emerg Med J 2004;21:746-7.

5. Lampert MB, Lang RM. Peripartum cardiomyopath. Am Heart J 1995;130:860-70.

6. Sliwa K, Forster O, Fitzgerald Z, et al. Outcome of subsequent pregnancy in patients with documented peripartum cardiomyopathy. Am J Cardiol 2004;93:1441-3.

7. Sibai BM. Diagnosis and management of gestational hypertension and preeclampsia. Obstet Gynecol 2003;102:181-92.

8. Benedetti TJ, Kates R, Williams V. Hemodynamic observations in severe preeclampsia complicated by pulmonary edema. Am J Obstet Gynecol 1985;152:330-4.

9. Sibai BM, Mabie BC, Harvey CJ, et al. Pulmonary edema in severe preeclampsia-eclampsia: analysis of thirty-seven consecutive cases. Am J Obstet Gynecol 1987;156:1174-9.

10. Clark SL, Hankins GD, Dudley DA, et al. Amniotic fluid embolism: analysis of the national registry. Am J Obstet Gynecol 1995;172:1158-67.

11. Huybrechts W, Jorens PG, Jacquemyn Y, et al. Amniotic fluid embolsim: a rare cause of acute left-sided heart failure. Acta Cardiol 2006;61:643-9.

12. Clark SL, Pavlova Z, Greenspoon J, et al. Squamous cells in the maternal pulmonary circulation. Am J Obstet Gynecol 1986; 154:104-6.

13. Vanmaele L, Noppen M, Vincken W, et al. Transient left heart failure in amniotic fluid embolism. Intensive Care Med 1990; 16:269-71.

14. Sciscione AC, Ivester T, Largoza M, et al. Acute pulmonary edema in pregnancy. Obstet Gynecol 2003;101:511-5.

Correspondence to: Dr. Antonio Meriano, Emergency Department, Windsor Regional Hospital, 1995 Lens Ave., Windsor ON N8W 1L9; tmeriano@gmail.com 